УДК 614.83:519.688

BAK: 05.26 .03

DOI: 10.32686/1812-5220-2018-15-74-79

ISSN 1812-5220

(C) Проблемы анализа риска, 2018

\section{Е. Ю. Колесников,} ФГБОУ ВО «Поволжский государственный технологический университет», г. Йошкар-Ола

\section{Интервальная оценка} неопределенности параметров аварийного и пожарного рисков

\begin{abstract}
Аннотация
Неопределенность является атрибутом пожарного и аварийного рисков, имеет как объективное, так и субъективное происхождение. Подобно измерительной неопределенности в современной метрологии, неопределенность в теории риска обладает качественным и количественным аспектами. Известно несколько способов ее количественной оценки. С учетом особенностей реальных опасных производственных объектов вероятностный подход к выполнению количественного оценивания неопределенности их параметров риска неприменим. Хорошей альтернативой является оценка аварийного и пожарного рисков в интервальной постановке. Помимо непосредственной оценки неопределенности полученных результатов - показателей риска — она позволяет выполнить оценку параметрической чувствительности используемых физико-математических моделей, а также оценить консервативность принятых допущений.
\end{abstract}

\title{
An interval assessment of the uncertainty of fire and emerging risk parameters
}

\section{E. Yu. Kolesnikov,}

Volga State University of Technology,

Yoshkar-Ola

\section{Annotation}

Uncertainty as an attribute of fire and emerging risks has both objective and subjective origin. Like measure uncertainty in modern metrology, the uncertainty in the risk theory has qualitative and quantitative aspects. Several methods for uncertainty quantifying are known. Taking into account the specifics of dangerous industrial objects, the probabilistic approach to quantitative estimation of their risk parameter uncertainty is inapplicable. A good alternative is to assess the fire and emerging risks in the interval setting. In addition to directly assessing the uncertainty of the results obtained - the risk indicators - it also makes it possible to estimate the parametric sensitivity of the model and to assess the conservatism of the assumptions made.

Keywords: fire, industrial safety, emerging and fire risks, uncertainty quantifying.

\section{Содержание}

Введение

1. Неопределенность параметров риска, ее типы и происхождение

2. Количественная оценка неопределенности

3. Преимущества интервальной постановки задачи

Заключение

Литература 


\section{Введение}

В последнее время наблюдается рост интереса отечественного научного сообщества к проблеме неопределенности, ее анализу и методам количественного оценивания. Это заметно по увеличению числа публикаций на русском языке по данной теме. При этом следует отметить, что на «международном» английском языке количество статей, монографий, отчетов и диссертаций по проблематике неопределенности исчисляется сотнями.

Понятие «неорределенность» наиболее часто используется для качественной оценки современных представлений о предмете исследования, оно характеризует их неполноту, неточность, неоднозначность. Нет никакого сомнения в том, что в науке оно появилось намного раньше, чем понятие «риск». Именно в таком, качественном смысле понятие «неопределенность» широко применяется в теории экономического (финансового, инвестиционного) риска. Однако именно теории аварийного, техногенного риска наука обязана новой трактовке неопределенности, в которой она приобрела количественный аспект. Прецедентом стал знаменитый отчет "WASH-1400" [1] о безопасности коммерческих ядерных реакторов, разработанный группой специалистов под началом проф. Расмуссена в 1975 г.

\section{1. Неопределенность параметров риска, ее типы и происхождение}

Теория анализа аварийного риска является научной дисциплиной, претендующей на роль теоретического базиса современной промышленной и пожарной безопасности. В своей методологии она стремится в максимальной мере соответствовать естественнонаучным стандартам. Однако сложность объектов ее исследования, неполнота, неточность (объективная или субъективная) исходных данных вынуждает при прогнозировании аварийных ситуаций вместо математического моделирования использовать экспертные суждения. А поскольку подобные методы выходят за рамки естествознания, это не позволяет многим специалистам относить данную методологию к области “hard science” (классической науки) [2].

Важнейшим этапом процедуры анализа аварийного (пожарного) риска является этап количествен- ной оценки риска (КОР). Целью КОР является получение количественных параметров риска, оценивающих промышленную (пожарную) безопасность исследуемого опасного объекта путем выполнения расчетов по регламентированным физико-математическим моделям и при определенном наборе входных параметров (часть из которых нередко задается экспертно). В настоящее время принято задавать величину этих параметров, равно как и параметров самой модели, точечными (скалярными) числами. Очевидно, что и результат расчета (показатель риска) в таком случае также оказывается точечным числом. Между тем при выполнении КОР величины подавляющей части входных параметров модели не являются скалярами, а могут быть заданы лишь некоторыми диапазонами, в пределах которых они имеют разброс.

Отчего же так происходит? Анализ показывает, что тому есть объективные и субъективные причины:

- с одной стороны, поскольку время начала прогнозируемой аварии заранее неизвестно (если это не диверсия), точно задать величину важнейших параметров задачи (метеорологические условия, энергетический потенциал аварийного выброса, местоположение персонала и подвижных объектов и т.д.) невозможно;

- с другой - используемые физико-математические модели аварийных процессов обладают ограниченной точностью, сведения о величинах их параметров неполны, неточны, они могут меняться в некоторых диапазонах.

Таким образом, неопределенность является атрибутом, т.е. неотъемлемым свойством любого параметра, характеризующего риск [3]. В этом заключается качественный аспект неопределенности.

Количественный аспект неопределенности в теории аварийного (пожарного) риска заключается в том, что она используется как количественная мера разброса - как величины входных параметров задачи, так и получаемого результата.

Таким образом, в силу вышеуказанных обстоятельств результаты КОР в подавляющем большинстве случаев будут являться некоторыми интервалами, характеризующими диапазон возможных значений, которые обоснованно могут быть им приписаны. Именно в пределах этих интервалов будет иметь место разброс их значений. Следовательно, 
показатель риска, выраженный скалярным числом, будет обладать скрытой неопределенностью, в ряде случаев - значительной.

В современной метрологии подобный интервал принято характеризовать величиной измерительной неопределенности. Таким образом, количественный аспект неопределенности в теории аварийного (пожарного) риска в целом соответствует трактовке неопределенности результатов измерений современной метрологией.

В метрологии при наличии достаточного массива экспериментальных данных интервал неопределенности задают бактором ошибки ЕF [4]:

$$
E F=\sqrt{\frac{\mathrm{G}_{0,95}}{\mathrm{G}_{0,05}}},
$$

где $\mathrm{G}_{0,95}$ и $\mathrm{G}_{0,05}$ - верхняя и нижняя границы $95 \%$ го доверительного интервала измеряемой величины G соответственно. Формула (1) показывает, что в метрологии используется классический, вероятностный подход к количественной оценке неопределенности (КОН).

Следует отметить, что уже в 80-х гг. прошлого века в Западной Европе понимали необходимость количественной оценки неопределенности при выполнении КОР. Так, например, Руководство по КОР CPR-12E [5] («красная» книга TNO) в качестве источников неопределенности рассматривает: а) допущения о распределении людей на территории зоны поражения; б) исключение из рассмотрения некоторых сценариев аварии; в) оценку вероятности токсического поражения человека по зависимостям «доза - эффект» и др.

Важнейшая для лучшего понимания природы неопределенности результатов КОР идея, повидимому, впервые была сформулирована в отчете МАГАТЭ № 100 [6] серии «Безопасность», подготовленном в 1989 г. Она заключалась в выделении двух типов неопределенности - объективной, стохастической (aleatory) и субъективной, познавательной, т. е. обусловленной состоянием знаний о предмете (epistemic). Первая обусловлена объективной изменчивостью, варьированием величины параметров, описывающих свойства опасного объекта и окружающей среды, вторая - недостатком сведений об истинной величине прочих параметров модели, их неточностью [2].
Еще один, значимый тип неопределенности терминологический (коммуникативный), связан с процессом оценивания экспертом аварийного (пожарного) риска, трактовки им полученных результатов. Эта неопределенность возникает изза того, что люди воспринимают любую информацию в соответствии со своими представлениями и установками.

Четвертый тип - вычислительная неорределенность. Результаты приближенных вычислений на ПЭВМ всегда обладают неопределенностью, которая аккумулирует неточности, обязанные своим появлением округлениям, отбрасыванию некоторых членов ряда и прочим особенностям этих методов. Известно, что интервальные расчеты порождают особый вид вычислительной неопределенности, которая может оказаться очень значительной, если не предпринимать специальных мер противодействия.

\section{2. Количественная оценка неопределенности}

Проблема количественной оценки неопределенности результатов КОР за рубежом впервые была поставлена в повестку дня в 1972 г., в самом начале работы над проектом “WASH-1400”, с тех пор там ведется интенсивный научный поиск путей ее решения. Для выполнения КОН используют качественные, полуколичественные и количественные методы. С классической точки зрения последние принято делить на вероятностные и детерминированные, хотя в последнее время предложен и ряд гибридных методов КОН.

Вероятностный подход основан на постулировании применимости вероятностных законов. Само понятие закона означает, в частности, устойчивость каких-то соотношений между параметрами. В случае вероятностных закономерностей это проявляется как статистическая устойчивость явлений, когда при увеличении объема выборки выборочное среднее, например, стремится к величине математического ожидания.

В силу ряда причин вероятностный способ КОН наиболее широко используется при выполнении КОР объектов ядерного цикла [3]. Именно на этот способ выполнения КОН ссылаются в своей статье [7] М.Н. Тихонов и М.И. Рылов. 
Свойства же реальных опасных производственных объектов таковы, что ни они сами, ни их технологические блоки не образуют генеральных совокупностей. Стало быть, статистической устойчивостью эти свойства не обладают. А это непосредственно означает, что условия применимости вероятностного подхода к выполнению $\mathrm{KOH}$ параметров аварийного риска подобных объектов отсутствуют.

Одной из альтернатив вероятностному способу $\mathrm{KOH} \mathrm{параметров} \mathrm{аварийного} \mathrm{риска} \mathrm{является} \mathrm{детер-}$ министический подход, подразумевающий оценку границ диапазона, в котором может меняться величина искомого параметра. Известны следующие методы детерминистического оценивания неопределенности: коэффициента неопределенности, граничных значений, интервальный. Важно подчеркнуть, что в детерминистической постановке оцениваются только границы диапазона неопределенности величины параметра, никаких утверждений или оценок о ее поведении внутри интервала не делается.

\section{3. Преимущества интервальной постановки задачи}

В последнее время набирает популярность решение инженерных задач в рамках интервального подхода, который подразумевает: 1) обоснованное задание значений входных параметров задачи интервалами; 2) выполнение всех необходимых вычислений методами интервального анализа. В итоге результат расчета также оказывается интервальным числом (интервалом), ширина которого непосредственно характеризует его неопределенность. Таким образом, интервальная постановка позволяет получить прямую количественную оценку неопределенности результатов расчета, что является ее безусловным преимуществом.

Следует отметить, что задание неопределенности величины параметра интервальным числом возможно только при выполнении условия «выпуклости», означающем, что она может принимать любые значения в пределах заданных границ.

Как уже было сказано, интервальные расчеты в классической (наивной) версии интервального анализа сопровождаются значительной неопределенностью, заключающейся в неоправданном увеличении ширины интервалов результатов расчетов. Этот эффект появляется из-за неоднократного вхождения любого интервального параметра в расчетное соотношение либо из-за наличия функциональной связи между входными параметрами расчетной модели. Для преодоления этого негативного эффекта в интервальном анализе разработаны и используются несколько методов - Рамона Мура, глобальной оптимизации, аффинное представление входных параметров [8].

Показано [9], что, наряду с собственно КОН, интервальная постановка выполнения КОР позволяет решить и ряд других важных для практики задач, например, оценить параметрическую чувствительность используемых физико-математических моделей. Для того чтобы сделать это, достаточно задать все независимые входные параметры модели, кроме исследуемого, очень узкими интервалами. Ширина интервала полученного результата (отклик модели) будет непосредственно характеризовать ее параметрическую чувствительность к исследуемому параметру.

Кроме того, интервальная постановка задачи дает возможность оценить консервативность принимаемых при моделировании допущений. Известно, что в первоначальный период развития методологии КОР часто рекомендовался так называемый консервативный подход, в рамках которого для расчета целевых показателей риска всем входным параметрам задачи присваивались их экстремальные значения - максимальные или минимальные (в зависимости от их влияния на величину целевого параметра риска).

Подобные точечные консервативные оценки легко могут быть выполнены в рамках интервального подхода. Для этого входным параметрам задачи следует приписать значения, соответствующие либо верхней, либо нижней границе их интервалов (в зависимости от влияния данного параметра на величину целевого параметра).

В нашей работе [9] на примере аварийного сценария «Объемный взрыв облака ТВС» выполнено сравнение использования консервативного подхода в традиционной точечной и интервальной постановках. Для этого сначала была получена точечная консервативная оценка избыточного давле- 


\begin{tabular}{|c|c|c|}
\hline Сценарий аварии, поражающий фактор & Интервальный расчет & $\begin{array}{l}\text { «Консервативный» } \\
\text { точечный расчет }\end{array}$ \\
\hline Пожар пролива, интенсивность падающего теплового потока I, кВт/м² & {$[7,7,14,4]$} & 14,4 \\
\hline Огненный шар, интенсивность падающего теплового потока I, кВт/м² & {$[9,6,65,3]$} & 65,3 \\
\hline Факельное горение струи, интенсивность падающего теплового потока I, кВт/м² & {$[16,2,18,2]$} & 18,2 \\
\hline Объемный взрыв ТВС, избыточное давление взрыва $\triangle \mathrm{P}, \mathrm{k}$ Па & {$[0,017,2,7]$} & 2,44 \\
\hline Пожар-вспышка, радиус зоны воздействия $\mathrm{R}_{\mathrm{F}}, \mathrm{M}$ & {$[1,3,158]$} & 122 \\
\hline
\end{tabular}

ния взрыва при наибольших значениях всех входных параметров, кроме НКПР бензина, которому было приписано минимальное значение 0,96\%. Полученная при этом точечная оценка прогнозируемого избыточного давления взрыва на удалении 100 м составила $\Delta \mathrm{P}=2,44$ кПа. Затем была выполнена консервативная оценка в интервальной постановке (с использованием метода глобальной оптимизации), которая дала интервальную оценку $\Delta \mathrm{P} \in[0,017,2,7]$ кПа. Заметно, что верхняя граница интервала $\Delta \mathrm{P}$ существенно превышает точечную «консервативную» оценку избыточного давления взрыва. Аналогичное сравнение точечных «консервативных» оценок поражающих факторов с их интервальными оценками было затем выполнено для четырех других сценариев аварии, полученные результаты отражены в таблице.

Анализ данной таблицы ясно показывает, что в двух из пяти рассмотренных сценариях аварии точечные «консервативные» значения целевых метрик оказались меньше верхних границ их интервальных оценок. Таким образом, «консервативные» точечные оценки поражающих факторов аварии оказались не вполне консервативными.

Это произошло оттого, что в общем случае используемые в модели математические функции не являются монотонными, а немонотонные функции достигают своих экстремальных значений необязательно на границах области определения. В то же время метод глобальной оптимизации в интервальной постановке позволяет гарантированно найти границы (min и $\max$ ) области значений целевой функции любого типа.

\section{Заключение}

В статье показано, что выполнение количественной оценки аварийного и пожарного рисков опасных производственных объектов в интервальной постановке в настоящее время является делом очень перспективным. Однако преимущества данного подхода могут быть реализованы только при условии подавления дополнительной неопределенности, присущей методам интервального анализа.

\section{Литература [References]}

1. U.S. Nuclear Regulatory Commission. Reactor Safety Study - An Assessment of Accident Risk in Commercial Nuclear Power Plants. WASH-1400 (NUREG-75/014). Main report, 1975.226 p.

2. Колесников Е.Ю. Количественное оценивание неопределенности техногенного риска. Часть 1 // Проблемы анализа риска. 2013. Т. 10. №2. С. 48-71. [Kolesnikov E.Yu. Quantitative estimation of the uncertainty of technogenic risk. Part 1 // Issue of risk analysis. 2013. Vol. 10. No 2. P. 48-71.]

3. Колесников Е.Ю. Количественное оценивание неопределенности техногенного риска. Часть 2 // Проблемы анализа риска. 2013. Т. 10. №3. С. 8-31. [Kolesnikov E.Yu. Quantitative estimation of the uncertainty of technogenic risk. Part 2 // Issue of risk analysis. 2013. Vol. 10. No 3. P. 8-31.]

4. JCGM 100:2008 GUM 1995 with minor corrections Evaluation of measurement data - Guide to the expression of uncertainty in measurement. $134 \mathrm{p}$.

5. CPR-12E Methods for determining and processing probabilities (Red book). 3-nd ed. Hague: VROM, 2005. $604 \mathrm{p}$. 
6. IAEA Safety series №100. Evaluating the reliability of prediction made using environmental transfer models IAEA, Vienna, 1989. 105 p.

7. Тихонов М.Н., Рылов М.И. После Чернобыля и Фукусимы-1: выявление и оценка неопределенностей и маловероятных рисков с катастрофическими последствиями // Проблемы анализа риска. 2014. T. 11. № 2. C. 24-49. [Tikhonov M.N., Rylov M.I. After Chernobyl and Fukushima-1:identification and evaluation of the uncertainties and unlikely risks with catastrophic consequences // Issue of risk analysis. Vol. 11. 2014. No2. P. 24-49.]

8. Колесников Е.Ю. Количественная оценка аварийного риска: анализ неопределенности // Безопасность труда в промышленности. 2018. №2. С. 64-70. [Kolesnikov E.Yu. Quantitative assessment of accident risk: uncertainty analysis // Occupational safety in industry. 2018. No 2. P. 64-70.]

9. Колесников Е.Ю., Теляков Э.Ш. Количественная оценка аварийного риска: оценка параметрической чувствительности моделей и консервативно- сти принятых допущений // Безопасность труда в промышленности. 2018. №3. С. 63-67. [Kolesnikov E.Yu., Telyakov E.Sh. Quantitative assessment of emergency risk: assessment of the parametric sensitivity of the models and the conservativeness of the accepted assumptions // Occupational safety in industry. 2018. No 3. P. 63-67.]

\section{Сведения об авторе}

Колесников Евгений Юрьевич: кандидат физико-математических наук, доцент кафедры безопасности жизнедеятельности Поволжского государственного технологического университета

Количество публикаций: более 80, в том числе монографий, учебников и учебных пособий - 9

Область научных интересов: методология анализа техногенного риска, количественная оценка неопределенности Контактная информачия:

Адрес: 424000, г. Йошкар-Ола, пл. Ленина, д. 3

Тел.: +7 (8362) 268-68-92

E-mail: e.konik@list.ru 